\title{
Utilização de ferramenta gráfıca para a interpretação de dados de EPMA na caracterização microestrutural de Ligas Ternárias à base de Nióbio
}

\section{Use of a graphical tool for the interpretation of EPMA data in micro- structural characterization of Niobium based Ternary Alloys}

\author{
1 João Carlos Jânio Gigolotti carlosjanio@uol.com.br \\ 1 Bruna Sousa Cardoso \\ 1 Ramon Durço Pereira \\ 2 Gilberto Carvalho Coelho \\ 2 Carlos Angelo Nunes \\ 3 Jean-Marc Joubert

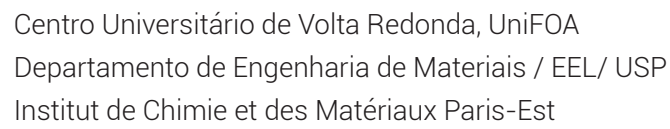

\section{Resumo}

O desenvolvimento de materiais para aplicação e aumento da eficiência de equipamentos submetidos a altas temperaturas de trabalho, como turbinas aeronáuticas, tem motivado o estudo de materiais à base de metais refratários, dentre os quais o nióbio, o cromo, o háfnio e o titânio. 0 conhecimento das relações de fases nesses materiais através da elaboração de diagramas de fases é fundamental para a determinação de suas propriedades mecânicas e térmicas. Com o objetivo de determinar a seção isotérmica a $1200{ }^{\circ} \mathrm{C}$ dos sistemas $\mathrm{Nb}-\mathrm{Si}-\mathrm{Ti}$ e $\mathrm{Cr}-\mathrm{Hf}-\mathrm{Nb}$, foi realizada a caracterização microestrutural de ligas tratadas termicamente a $120{ }^{\circ} \mathrm{C}$ por 720 horas, utilizando-se de difratometria de raios- $X$, imagens de microscopia eletrônica de varredura e análise em microssonda eletrônicade forma conjugada. $\mathrm{O}$ objetivo deste artigo é apresentar uma forma de analisar os dados deEPMAcom a utilização de ferramenta gráfica. Como resultado, foi elaborada uma proposta para a seção isotérmica a $1200^{\circ} \mathrm{C}$ do sistema $\mathrm{Nb}$-Si-Ti, que apresenta oito campos de equilíbrio trifásico, e outra proposta para a seção isotérmica a $1200{ }^{\circ} \mathrm{C}$ do sistema $\mathrm{Cr}-\mathrm{Hf}-\mathrm{Nb}$, que apresenta um campo de equilíbrio trifásico. Ficou evidenciada a validade e a eficiência da utilização de ferramenta gráficapara a interpretação de resultados de EPMA.

\section{Palavras-chave:}

Ligas de Nióbio. Seção isotérmica. EPMA.

\begin{abstract}
The development of materials for application and increased efficiency of equipment submitted to high work temperature, such as aeronautical turbines, has motivated the study of materials based on refractory metals, including niobium, chromium, hafnium and titanium. The knowledge of the phase relations in these materials through phase diagrams is fundamental for the determination of their mechanical and thermal properties. In order to determine the isothermal section at $1200{ }^{\circ} \mathrm{C}$ of the $\mathrm{Nb}-\mathrm{Si}-\mathrm{Ti}$ and $\mathrm{Cr}-\mathrm{Hf}-\mathrm{Nb}$ systems, a detailed microstructural characterization of heat treated alloys at $1200^{\circ} \mathrm{C}$ for 720 hours was carried out, using $X$-ray diffractometry, scanning electron microscopy and electron microprobe analysis in a conjugated form. The purpose of this paper is to present a way of analyzing EPMA data with the use of a graphical tool, which facilitates their interpretation. As a result, one proposal for the $1200^{\circ} \mathrm{C}$ isothermal section of the $\mathrm{Nb}$ Si-Ti system was elaborated, that shows eight threephase equilibrium fields, and another proposal for the $1200{ }^{\circ} \mathrm{C}$ isothermal section of the $\mathrm{Cr}-\mathrm{Hf}-\mathrm{Nb}$ system, that shows just one three-phase equilibrium field. The validity and the efficiency of the use of a graphic tool for the interpretation of EPMA results were evidenced.
\end{abstract}

\section{Keywords:}

Niobium alloys. Isothermal section. EPMA.

\section{Como você deve citar?}

GIGOLOTTI, João Carlos Jânio et. al. Utilização de Ferramenta Gráfica para a Interpretação de Dados de EPMA na Caracterização Microestrutural de Ligas Ternárias à base de Nióbio. Cadernos UniFOA, Volta Redonda, n. 40, p. 4155, agosto 2019. 
Utilização de ferramenta gráfica para a interpretação de dados de EPMA

na caracterização microestrutural de Ligas Ternárias à base de Nióbio

\section{INTRODUÇÃO}

Os problemas típicos de engenharia envolviam, até meados do século XX, em grande parte, soluções técnicas adequadas e minimização de custos com restrita ênfase na solução de problemas sociais e, menos ainda, ambientais. A expansão dos princípios democráticos e dos direitos humanos a partir do final da Segunda Guerra Mundial (1945), acelerada com o final da Guerra Fria (1989), aliada à definição de princípios básicos e acordos internacionais para implantar medidas para a preservação ambiental e controle da emissão de poluentes, a partir da primeira Conferência Mundial sobre o Meio Ambiente (United Nations Conference on the Human Environment), em Estocolmo (1972), começaram a alterar aquela equação.

No que tange aos meios de transporte, a diminuição da emissão de poluentes passa, necessariamente, pelo aumento da eficiência dos motores à combustão interna e das turbinas aeronáuticas e a gás. No caso específico das turbinas, o desenvolvimento de ligas à base de metais refratários e outros componentes, capazes de atender aos requisitos operacionais a elevadas temperaturas, acima de 1000 ${ }^{\circ} \mathrm{C}$, tem sido largamente estudado [1,2,3,4]. Pesquisas têm demonstrado que compostos à base de $\mathrm{Nb}$ Si são adequados para a aplicação em turbinas aeronáuticas [5-8]. Em acréscimo, a adição de $\mathrm{Ti}, \mathrm{Cr}$, $\mathrm{Hf}, \mathrm{B} \mathrm{e} \mathrm{Al} \mathrm{incrementaria} \mathrm{as} \mathrm{propriedades} \mathrm{desses} \mathrm{materiais} \mathrm{[9-14].} \mathrm{Ti} \mathrm{e} \mathrm{Al,} \mathrm{particularmente,} \mathrm{aumentam} \mathrm{a}$ resistência à oxidação à alta temperatura em compostos de $\mathrm{Nb}-\mathrm{Nb}_{5} \mathrm{Si}_{3}$.

Dentro desse contexto, Gigolotti et al. $[15,16]$ realizaram a determinação experimental e elaboraram propostas para a seção isotérmica a $1200^{\circ} \mathrm{C}$ do sistema $\mathrm{Nb}$-Si-Ti [15] e do sistema $\mathrm{Cr}-\mathrm{Hf}-\mathrm{Nb}$ [16]. Para tal, foram muito importantes dados obtidos por ElectronProbeMicroanalyzer (EPMA).

Este trabalho tem por objetivo apresentar um método de interpretação de dados de EPMA, com a utilização de ferramenta gráfica, para a caracterização microestrutural de ligas metálicas, especificamente ligas compostas de Nióbio.

Há diversos estudos que resultaram em propostas para o sistema $\mathrm{Nb}$-Si-Ti, quer para a projeção liquidus quer para seções isotérmicas a diferentes temperaturas, tanto realizados por modelagem computacional [17-19] quanto por meio de diferentes procedimentos experimentais [20-27]. Apesar da grande quantidade de propostas existentes, apenas duas apresentaram a seção isotérmica a 1200 ${ }^{\circ} \mathrm{C}$ do sistema $\mathrm{Nb}$-Si-Tia partir de dados experimentais, uma elaborada por Wang et al. [23] e outra por Zhao et al. [24]. Embora ambos os estudos tivessem por base metodológica a utilização de pares de difusão, as propostas apresentadas foram bem discrepantes no que tange às regiões de equilíbrio trifásico, Figuras 1a e 1b.Por sua vez, a proposta de Gigolotti et al. para a seção isotérmica a $1200^{\circ} \mathrm{C}$ do sistema $\mathrm{Nb}-\mathrm{Si}$-Ti [15], Figura 2a e Tabela 1a, mostrou a existência de oito campos de equilíbrio trifásico: aNb(Ti) $)_{5} \mathrm{Si}_{3}-(\mathrm{Nb}, \mathrm{Ti})_{3} \mathrm{Si}-(\mathrm{Nb}, \mathrm{Ti})_{s S^{\prime}} \mathrm{Ti}(\mathrm{Nb})_{5} \mathrm{Si}_{3}-(\mathrm{Nb}, \mathrm{Ti})_{3} \mathrm{Si}-(\mathrm{Nb}, \mathrm{Ti})_{s S^{\prime}}$ aNb$(\mathrm{Ti})_{5} \mathrm{Si}_{3}-(\mathrm{Nb}, \mathrm{Ti})_{3} \mathrm{Si}-\mathrm{Ti}(\mathrm{Nb})_{5} \mathrm{Si}_{3^{\prime}}$ $\mathrm{aNb}(\mathrm{Ti})_{5} \mathrm{Si}_{3}-\mathrm{Ti}(\mathrm{Nb})_{5} \mathrm{Si}_{4}-\mathrm{Ti}(\mathrm{Nb})_{5} \mathrm{Si}_{3^{\prime}} \mathrm{aNb}_{5}(\mathrm{Ti}) \mathrm{Si}_{3}-\mathrm{Ti}(\mathrm{Nb})_{5} \mathrm{Si}_{4}-\mathrm{Nb}(\mathrm{Ti}) \mathrm{Si}_{2^{\prime}} \mathrm{Ti}(\mathrm{Nb}) \mathrm{Si}-\mathrm{Ti}(\mathrm{Nb})_{5} \mathrm{Si}_{4}-\mathrm{Nb}(\mathrm{Ti}) \mathrm{Si}_{2^{\prime}}, \mathrm{Ti}(\mathrm{Nb})$ $\mathrm{Si}-\mathrm{Ti}(\mathrm{Nb}) \mathrm{Si}_{2}-\mathrm{Nb}\left(\mathrm{Ti}_{\mathrm{i}} \mathrm{Si}_{2} \mathrm{eSi}_{\mathrm{SS}}-\mathrm{Ti}(\mathrm{Nb}) \mathrm{Si}_{2}-\mathrm{Nb}(\mathrm{Ti}) \mathrm{Si}_{2}\right.$.

Por outro lado, não foram encontradas propostas para a seção isotérmica a $1200^{\circ} \mathrm{C}$ do sistema $\mathrm{Cr}-\mathrm{Hf}-\mathrm{Nb}$. Fujita et al. [28] apresentaram uma proposta experimental para a seção isotérmica a $1300^{\circ} \mathrm{C}$ limitada à região rica em $\mathrm{Cr}$ até a seção pseudo-binária $\mathrm{Cr}_{2} \mathrm{Hf}-\mathrm{Cr}_{2} \mathrm{Nb}$, Figura 1c, no contexto do estudo das propriedades mecânicas da fase tipo Laves C15. A proposta de Gigolotti et al. [16] para a seção isotérmica a $1200^{\circ} \mathrm{C}$ do sistema $\mathrm{Cr}-\mathrm{Hf}-\mathrm{Nb}$, Figura $2 \mathrm{~b}$ e Tabela $1 \mathrm{~b}$, mostrou as regiões monofásicas $\mathrm{aHf}_{s s^{\prime}}(\beta \mathrm{Hf}, \mathrm{Nb})_{s S^{\prime}} \mathrm{a}\left(\mathrm{Cr}_{2} \mathrm{Nb}, \mathrm{Cr}_{2} \mathrm{Hf}\right)$ e $\mathrm{Cr}_{s S^{\prime}}$ as regiões de equilíbrio bifásico $\mathrm{aHf}_{s s}-\mathrm{a}\left(\mathrm{Cr}_{2} \mathrm{Nb}, \mathrm{Cr}_{2} \mathrm{Hf}\right),(\beta \mathrm{Hf}, \mathrm{Nb})$ $\mathrm{ss}^{-} \mathrm{a}\left(\mathrm{Cr}_{2} \mathrm{Nb}, \mathrm{Cr}_{2} \mathrm{Hf}\right), \mathrm{aHf}_{s s}-(\beta \mathrm{Hf}, \mathrm{Nb})_{s s}$ e $\mathrm{Cr}_{s s}-\mathrm{a}\left(\mathrm{Cr}_{2} \mathrm{Nb}, \mathrm{Cr}_{2} \mathrm{Hf}\right)$ e a região de equilíbrio trifásico $\mathrm{aHf}_{s s}-\mathrm{a}(\mathrm{Cr}-$ $\left.{ }_{2} \mathrm{Nb}, \mathrm{Cr}_{2} \mathrm{Hf}\right)-(\beta \mathrm{Hf}, \mathrm{Nb})_{s S}$. 
Figura 1 - Propostas experimentais para seções isotérmicas de sistemas ternários à base de $\mathrm{Nb}$ seções a $1200{ }^{\circ} \mathrm{C}$ do sistema Nb-Si-Ti: (a) Wang et al. [23]; (b) Zhao et al. [24]; e (c) seção a $1300{ }^{\circ} \mathrm{C}$ do sistema Cr-Hf-Nb de Fujita et al. [28] (redesenhadas).

(a)
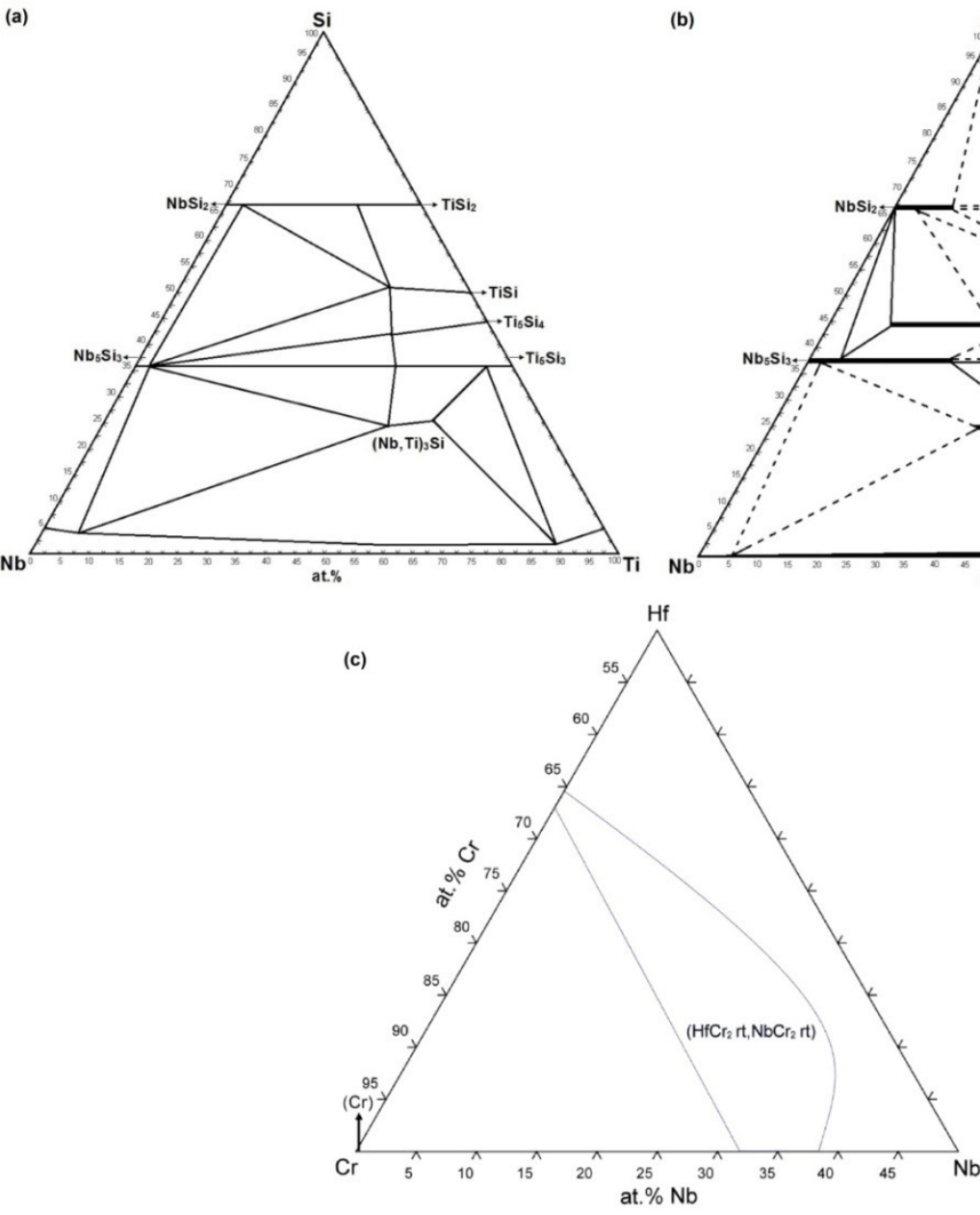

Fonte: Dos autores 2019.

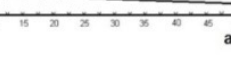

(b)

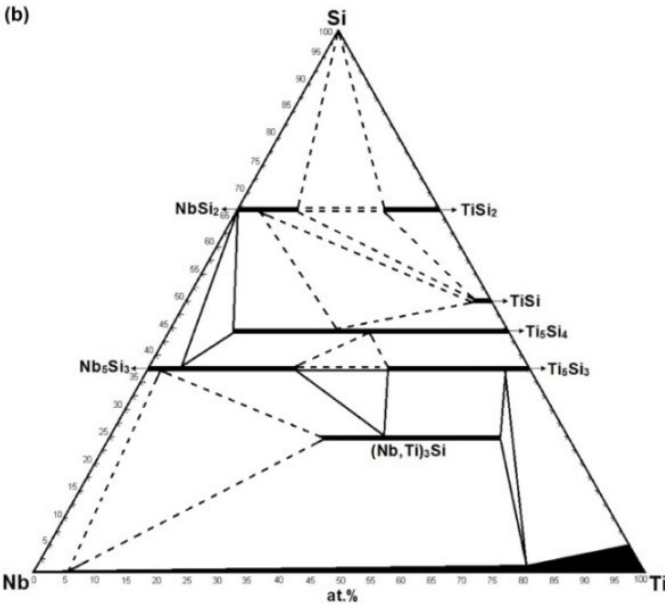


Figura 2 - Propostas experimentais de Gigolotti et al. para a seção isotérmica a $1200^{\circ} \mathrm{C}$ dos sistemas ternários à base de nióbio: (a) sistema Nb-Si-Ti [15]; (b) sistema Cr-Hf-Nb [16] (• composição nominal da liga; $\Delta$ composição da fase - posições aproximadas).

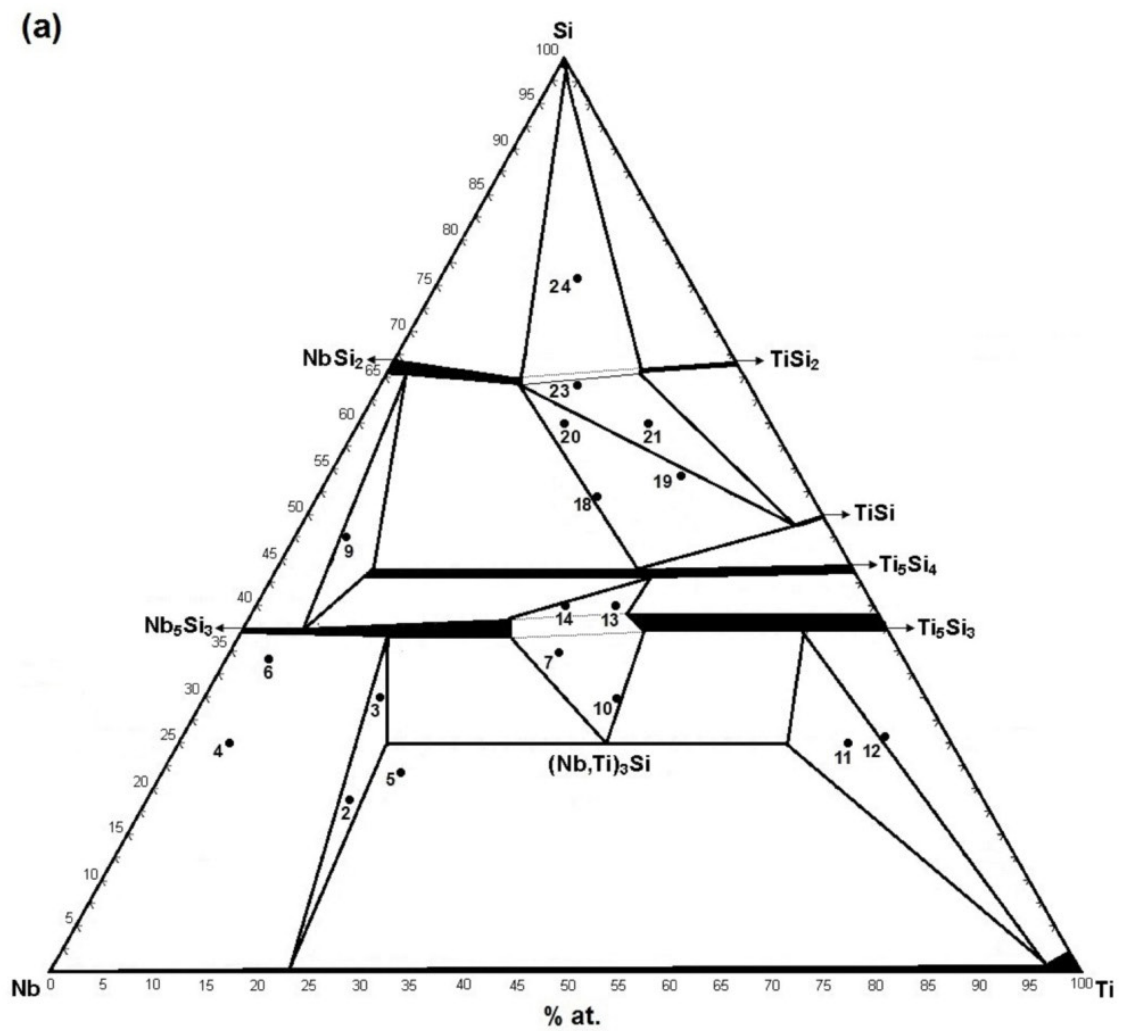

(b)

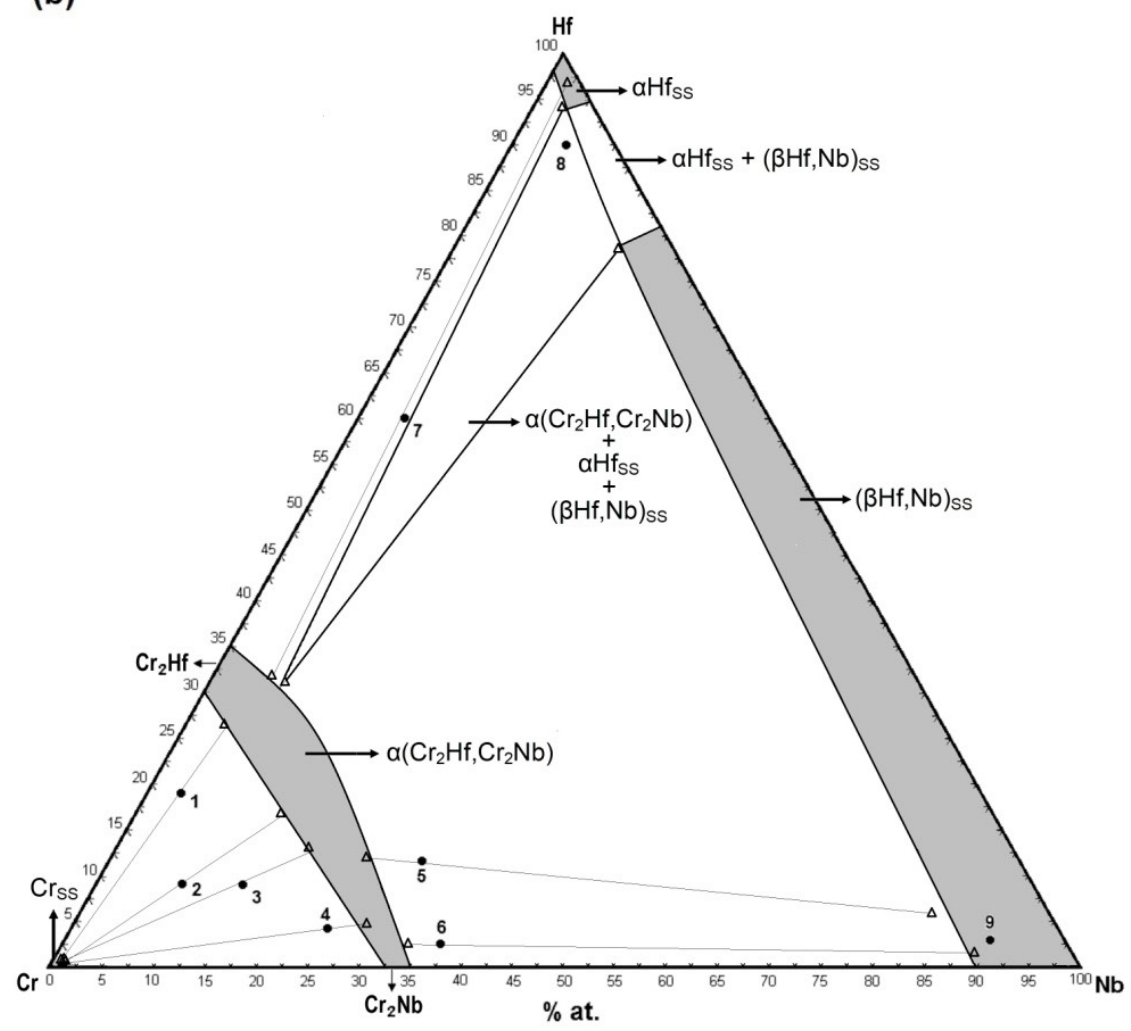

Fonte: Dos autores 2019. 
Tabela 1 - Campos de equilíbrio de seções isotérmicas a $1200^{\circ} \mathrm{C}$ de sistemas ternários à base de $\mathrm{Nb}$ definidos por Gigolotti et al.: (a) sistema Nb-Ti-Si [15]; (b) sistema Cr-Hf-Nb [16].

\begin{tabular}{|c|c|c|c|c|c|c|c|c|c|}
\hline \multicolumn{5}{|c|}{ (a) Sistema Nb-Ti-Si [15] } & \multicolumn{5}{|c|}{ (b) Sistema Cr-Hf-Nb [16] } \\
\hline \multirow{2}{*}{$\begin{array}{c}\text { Ligas HT } \\
1200{ }^{\circ} \mathrm{C} \\
720 \mathrm{~h}\end{array}$} & \multirow{2}{*}{$\begin{array}{c}\text { Campo } \\
\text { de equilí- } \\
\text { brio }\end{array}$} & \multicolumn{3}{|c|}{$\begin{array}{c}\text { Composição aproximada da fase } \\
\text { (\% at.) }\end{array}$} & \multirow{2}{*}{$\begin{array}{c}\text { Ligas HT } \\
1200^{\circ} \mathrm{C} \\
720 \mathrm{~h}\end{array}$} & \multirow[t]{2}{*}{$\begin{array}{l}\text { Campo de } \\
\text { equilíbrio }\end{array}$} & \multicolumn{3}{|c|}{$\begin{array}{c}\text { Composição aproximada da fase } \\
\text { (\% at.) }\end{array}$} \\
\hline & & $\mathrm{Nb}$ & $\mathrm{Ti}$ & Si & & & $\mathrm{Cr}$ & $\mathrm{Hf}$ & $\mathrm{Nb}$ \\
\hline \multirow[t]{3}{*}{2,3} & $\begin{array}{c}\mathrm{aNb}(\mathrm{Ti})- \\
\mathrm{Si}\end{array}$ & 49,4 & 14,1 & 36,5 & 1 & $\mathrm{Cr}_{s s}$ & 99,4 & 0,4 & 0,2 \\
\hline & $(\mathrm{Nb}, \mathrm{Ti})_{3} \mathrm{Si}$ & 54,4 & 20,6 & 25,0 & & $\begin{array}{l}\mathrm{a}\left(\mathrm{Cr}_{2} \mathrm{Nb},-\right. \\
\left.\mathrm{Cr}_{2} \mathrm{Hf}\right)\end{array}$ & 70,0 & 26,3 & 3,7 \\
\hline & $(\mathrm{Nb}, \mathrm{Ti})_{s s}$ & 75,7 & 23,6 & 0,7 & 2 & & 98,2 & 0,5 & 1,4 \\
\hline \multirow[t]{3}{*}{11,12} & $\mathrm{Ti}(\mathrm{Nb})_{5} \mathrm{Si}_{3}$ & 8,7 & 54,8 & 36,5 & & & 69,1 & 17,0 & 13,9 \\
\hline & $(\mathrm{Nb}, \mathrm{Ti})_{3} \mathrm{Si}$ & 16,3 & 58,9 & 24,8 & 3 & & 98,5 & 0,4 & 1,1 \\
\hline & $(\mathrm{Nb}, \mathrm{Ti})_{s s}$ & 3,5 & 95,6 & 0,9 & & & 68,6 & 13,0 & 18,4 \\
\hline \multirow[t]{3}{*}{7,10} & $\begin{array}{l}\mathrm{aNb}(\mathrm{Ti})- \\
{ }_{5} \mathrm{Si}_{3}\end{array}$ & 37,5 & 26,0 & 36,5 & 4 & & 99,0 & 0,1 & 0,9 \\
\hline & $(\mathrm{Nb}, \mathrm{Ti})_{3} \mathrm{Si}$ & 33,4 & 41,6 & 25,0 & & & 68,0 & 4,4 & 27,6 \\
\hline & $\mathrm{Ti}(\mathrm{Nb})_{5} \mathrm{Si}_{3}$ & 25,0 & 39,0 & 36,0 & 5 & $(\beta H f, N b)_{s s}$ & 11,6 & 5,3 & 83,1 \\
\hline \multirow[t]{3}{*}{13,14} & $\begin{array}{l}\mathrm{aNb}(\mathrm{Ti})- \\
{ }_{5} \mathrm{Si}_{3}\end{array}$ & 35,5 & 26,0 & 38,5 & & $\begin{array}{l}\mathrm{a}\left(\mathrm{Cr}_{2} \mathrm{Nb},-\right. \\
\left.\mathrm{Cr}_{2} \mathrm{Hf}\right)\end{array}$ & 63,6 & 12,0 & 24,4 \\
\hline & $\mathrm{Ti}(\mathrm{Nb})_{5} \mathrm{Si}_{4}$ & 19,7 & 37,2 & 43,1 & 6 & & 9,7 & 1,4 & 88,9 \\
\hline & $\mathrm{Ti}(\mathrm{Nb})_{5} \mathrm{Si}_{3}$ & 25,0 & 36,0 & 39,0 & & & 64,2 & 2,5 & 33,3 \\
\hline \multirow[t]{3}{*}{9} & $\begin{array}{c}\mathrm{aNb}_{5}(\mathrm{Ti}) \\
\mathrm{Si}_{3}\end{array}$ & 56,9 & 5,6 & 37,5 & 7 & $\mathrm{aHf}_{\mathrm{sS}}$ & 0,4 & 97,1 & 2,5 \\
\hline & $\mathrm{Ti}(\mathrm{Nb})_{5} \mathrm{Si}_{4}$ & 46,8 & 9,3 & 43,9 & & $\begin{array}{l}\mathrm{a}\left(\mathrm{Cr}_{2} \mathrm{Nb},-\right. \\
\left.\mathrm{Cr}_{2} \mathrm{Hf}\right)\end{array}$ & 63,4 & 31,9 & 4,7 \\
\hline & $\mathrm{Nb}(\mathrm{Ti}) \mathrm{Si}_{2}$ & 32,8 & 2,1 & 65,1 & 8 & $(\beta \mathrm{Hf}, \mathrm{Nb})_{s s}$ & 5,3 & 78,6 & 16,1 \\
\hline 18,19 & Ti(Nb)Si & 2,8 & 48,8 & 48,4 & & $\mathrm{aHf}_{s s}$ & 2,7 & 94,6 & 2,7 \\
\hline 20 & $\mathrm{Ti}(\mathrm{Nb})_{5} \mathrm{Si}_{4}$ & 21,0 & 35,6 & 43,4 & & $\begin{array}{c}\mathrm{a}\left(\mathrm{Cr}_{2} \mathrm{Nb},-\right. \\
\left.\mathrm{Cr}_{2} \mathrm{Hf}\right)\end{array}$ & Cr62,0 & 31,5 & 6,5 \\
\hline \multirow{4}{*}{21,23} & $\mathrm{Nb}(\mathrm{Ti}) \mathrm{Si}_{2}$ & 22,3 & 13,2 & 64,5 & 9 & $(\beta \mathrm{Hf}, \mathrm{Nb})_{\mathrm{sS}}$ & $\mathrm{Cr} 4,6$ & 3,8 & 91,6 \\
\hline & Ti(Nb)Si & 3,6 & 47,6 & 48,8 & & & & & \\
\hline & $\mathrm{Ti}(\mathrm{Nb}) \mathrm{Si}_{2}$ & 10,7 & 24,1 & 65,2 & & & & & \\
\hline & $\mathrm{Nb}(\mathrm{Ti}) \mathrm{Si}_{2}$ & 21,9 & 13,2 & 64,9 & & & & & \\
\hline \multirow[t]{3}{*}{24} & $\mathrm{Si}_{\mathrm{ss}}$ & 0,5 & 0,7 & 98,8 & & & & & \\
\hline & $\mathrm{Ti}(\mathrm{Nb}) \mathrm{Si}_{2}$ & 10,7 & 24,1 & 65,2 & & & & & \\
\hline & $\mathrm{Nb}\left(\mathrm{Ti} \mathrm{Si}_{2}\right.$ & 21,9 & 13,2 & 64,9 & & & & & \\
\hline
\end{tabular}


Utilização de ferramenta gráfica para a interpretação de dados de EPMA

na caracterização microestrutural de Ligas Ternárias à base de Nióbio

\section{PROCEDIMENTO EXPERIMENTAL}

O mesmo procedimento experimental foi utilizado para a avaliação de ambos os sistemas, $\mathrm{Nb}-\mathrm{Si}-\mathrm{Ti}$ e $\mathrm{Cr}-\mathrm{Hf}-\mathrm{Nb}$. As ligas com diferentes composições foram preparadas a partir de matérias-primas de alta pureza (> $99.9 \%$ wt.). Pedaços de Nb, Si, Ti e $\mathrm{Cr}, \mathrm{Hf}, \mathrm{Nb}$, num caso e noutro, foram pesados conforme a composição da liga, fundidos em forno a arco, em cadinho de cobre eletrolítico resfriado a água, com eletrodo de tungstênio não consumível, tendo sido usado getter de titânio para a remoção de $\mathrm{O}_{2} / \mathrm{H}_{2} \mathrm{O} /$ $\mathrm{N}_{2}$ residual e atmosfera de argônio. Cada amostra com 3-4 g foi fundida ao menos cinco vezes para garantir sua homogeneidade. Nesse processo a perda de massa foi desprezível.

O tratamento térmico das ligas foi realizado num forno tubular, a $1200^{\circ} \mathrm{C}$ durante $720 \mathrm{~h}$. Para o tratamento, as ligas fundidas foram acomodadas separadamente em tubos de quartzo preenchidos com argônio e com getter de Zr.

As ligas foram preparadas para caracterização por difratometria de raios-X (DRX), por microscópio eletrônico de varredura, no modo elétrons retroespalhados (MEV/IERE) e por EPMA. Para caracterização por DRX, pedaços das ligas foram triturados em pilão de aço e ágata até a obtenção de pó fino passante em peneira de 80 Mesh. Para a obtenção das imagens de MEV/IERE e dos dados de EPMA, pedaços das ligas foram embutidos em resina a frio e passadas por processo tradicional de metalografia, lixadas (\# 320-1200) e polidas com pasta de diamante.

As ligas foram caracterizadas da seguinte forma: (1) por DRX foram identificadas as fases presentes tendo por comparação modelos de difração construídos com o programa PowderCell for Windows (versão 2.4) [29], tendo por base dados cristalográficos compilados por Villars et Calvert [30]; (2) os resultados de DRX foram submetidos a refinamento pelo método de Rietveld [31] com o uso do programa FullProfSuite [32] para determinação da quantidade percentual de cada fase ${ }^{(a)}$; (3) tendo por base as fases identificadas nos DRX, as imagens obtidas em MEV/IERE foram caracterizadas considerando-se o contraste entre as fases corresponde à variação de tons de cinza de acordo com a massa molar da fase (> massa mais claro); (4) a análise conjuminada das imagens MEV/IERE com as imagens e dados obtidos por EPMA num instrumento CAMECA SX 100 [33] permitiram avaliar as microestruturas e as relações entre as fases que formam os campos de equilíbrio na seção isotérmica a $1200^{\circ} \mathrm{C}$; (5) os dados de EPMA, consolidados numa planilha eletrônica, permitiram determinar as composições aproximadas das fases que formam aqueles campos de equilíbrio, locar graficamente os pontos correlatos no diagrama de Gibbs e calcular a quantidade percentual de cada fase utilizando-se a regra da alavanca [34] ${ }^{(b)}$; e (6) os valores obtidos em (a) e (b) foram comparados para verificar a necessária semelhança dos resultados.

\section{UTILIZAÇÃO DE FERRAMENTA GRÁFICA PARA ANÁLISE DOS RESULTADOS DE EPMA}

As microssondas eletrônicas $[33,35]$ são instrumentos capazes de determinar, de forma não destrutiva, a composição elementar de uma amostra, em termos qualitativos e quantitativos, ou seja, a distribuição das concentrações elementares em microvolumes de superfícies de amostras irradiadas com um feixe de elétrons altamente concentrado, que leva as várias espécies elementares presentes a emitir raios- $X$ característicos. A composição da amostra pode ser facilmente identificada através da gravação de espectros em WDS (Wavelength Dispersive Spectroscopy). Os espectrômetros WDS são baseados na lei de Bragg e usam vários monocristais móveis tipo monocromadores. É a técnica de microanálise mais precisa disponível para todos os elementos químicos do boro ao urânio. 
O instrumento CAMECA SX 100 [33], que foi utilizado na avaliação dos sistemas $\mathrm{Nb}$-Si-Ti e Cr$\mathrm{Hf}-\mathrm{Nb}$, é equipado com ferramentas de microscopia que permitem a obtenção, simultaneamente, de dados de WDS e EDS (Energy Dispersive Spectroscopy), e imagens de MEV/IERE, com ampliação de imagem variando de 40 a 400.000 vezes.

A seguir, são apresentados dois exemplos daforma como foi usada ferramenta gráfica para a interpretação dos dados de EPMA, o primeiro referente a uma liga de $\mathrm{Nb}$-Si-Ti e o segundo referente a uma liga de $\mathrm{Cr}-\mathrm{Hf}-\mathrm{Nb}$.

Exemplo 1 - Análise dos dados de EPMA da Liga $24 \mathrm{HT}\left(\mathrm{Nb}_{11} \mathrm{Si}_{76} \mathrm{Ti}_{13} \%\right.$ at.), Figura 2a e Tabela $1 \mathrm{a}$, do sistema ternário $\mathrm{Nb}-\mathrm{Si}-\mathrm{Ti}$.

Os resultados de DRX da Liga $24 \mathrm{HT}$ tratada termicamente (heattreated - HT), Figura 3a, mostraram a presença das fases $\mathrm{Si}_{S S}$ (solução sólida), $\mathrm{NbSi}_{2}$ e TiSi ${ }_{2}$. As fases $\mathrm{NbSi}_{2}$ e $\mathrm{Si}_{S S}$ apresentam picos intensos enquanto a fase $\mathrm{TiSi}_{2}$ apresenta alguns picos de menor intensidade.

Tendo por base esses resultados foi identificada na imagem MEV/IERE da liga $\mathrm{Nb}_{11} \mathrm{Si}_{76} \mathrm{Ti}_{13} \%$ at., Figura $3 \mathrm{~b}$, uma microestrutura mista das fases $\mathrm{Si}_{\mathrm{SS}} \mathrm{eNb}(\mathrm{Ti}) \mathrm{Si}_{2} \mathrm{e} \mathrm{Ti}(\mathrm{Nb}) \mathrm{Si}_{2^{\prime}}$ o que caracteriza o campo de equilíbrio trifásico $\mathrm{Si}_{\mathrm{SS}}+\mathrm{Nb}(\mathrm{Ti}) \mathrm{Si}_{2}+\mathrm{Ti}(\mathrm{Nb}) \mathrm{Si}_{2}$. Observa-se uma correlação entre a grande quantidade de picos intensos das fases $\mathrm{Si}_{S S}$ e $\mathrm{Nb}\left(\mathrm{Ti}_{\mathrm{T}} \mathrm{Si}_{2}\right.$ no DRX e sua grande quantidade na micrografia, ao passo que a fase $\mathrm{Ti}(\mathrm{Nb}) \mathrm{Si}_{2}$ apresenta pequena quantidade na micrografia em consonância com o resultado de DRX.

Os resultados de EPMA da Liga $24 \mathrm{HT}\left(\mathrm{Nb}_{17} \mathrm{Si}_{76} \mathrm{Ti}_{13} \%\right.$ at.) foram lançados numa planilha eletrônica, como visto parcialmente na Figura 4a. Cada ponto medido traz sua composição em \% at. tanto para o $\mathrm{Nb}$ quanto para o Ti e o Si. Como exemplo, o ponto identificado como 10/1. mostra a seguinte composição em \% at.: Nb0,54333333, Ti 0,81333333 e Si 98,6533333.

Figura 3 - Resultados de caracterização de liga Nb-Si-Ti tratada termicamente a $1200{ }^{\circ} \mathrm{C}$ : (a) DRX da liga $24 \mathrm{HT}\left(\mathrm{Nb}_{11} \mathrm{Si}_{76} \mathrm{Ti}_{13}\right)$; (b) imagem MEV/IERE da liga $24 \mathrm{HT}\left(\mathrm{Nb}_{11} \mathrm{Si}_{76} \mathrm{Ti}_{13}\right)$.
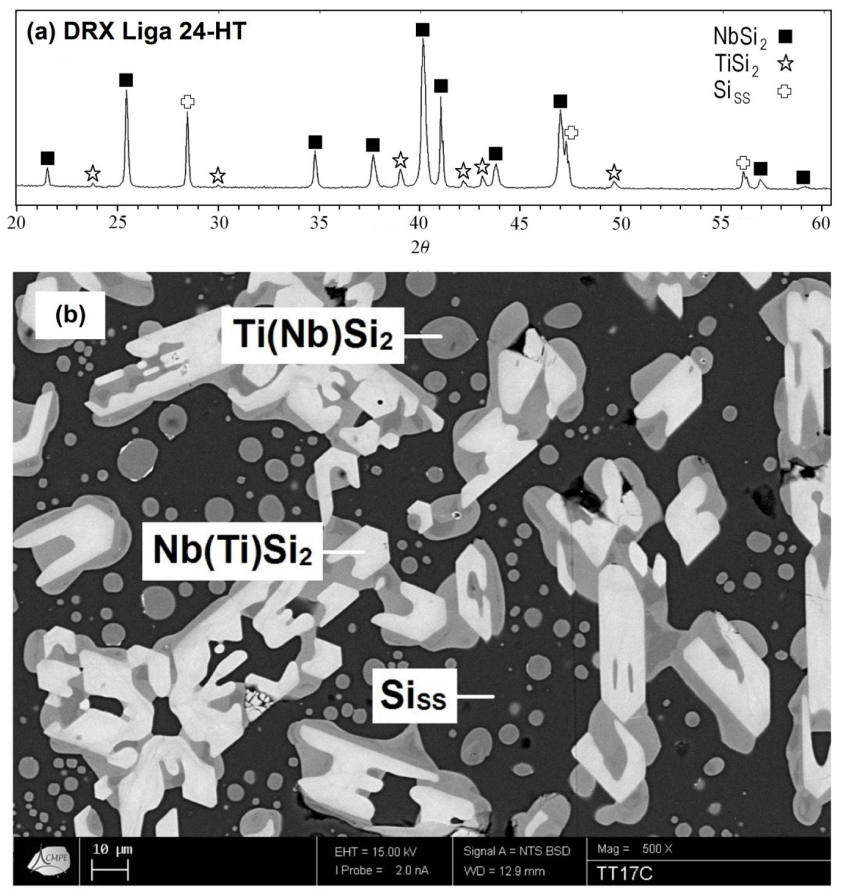

Fonte: Dos autores 2019 
Utilização de ferramenta gráfica para a interpretação de dados de EPMA

na caracterização microestrutural de Ligas Ternárias à base de Nióbio

A partir dos resultados de EPMA lançados na planilha eletrônica, cada ponto medido foi correlacionado a uma das três fases identificadas por DRX e em MEV, conforme atendimento a um dos seguintes critérios:

(a) 0 ponto medido está na fase $\mathrm{Nb}(\mathrm{Ti}) \mathrm{Si}_{2}$ se apresenta: $66,7 \%$ at. Si (até 2 und. \% at. para +/-), $>17,0 \%$ at. $\mathrm{Nb}$ e $<17,0 \%$ at. Ti;

(b) 0 ponto medido está fase $\mathrm{Ti}(\mathrm{Nb}) \mathrm{Si}_{2}$ se apresenta: $66,7 \%$ at. Si (até 2 und. \% at. para $+/-$ ), $>17,0 \%$ at. Ti e $<17,0 \%$ at. $\mathrm{Nb}$; e

(c) 0 ponto medido está fase $\mathrm{Si}_{\mathrm{SS}}$ se apresenta: $>98,0 \%$ at. $\mathrm{Si},<1,0 \%$ at. $\mathrm{Nb}$ e $<1,0 \%$ at. Ti.

Assim sendo, o ponto identificado como 10/1., no exemplo, conforme sua composição em \% at., preenche os critérios estabelecidos na letra (c) e, portanto, corresponde à fase $\mathrm{Si}_{S S}$.

Figura 4 - Resultados de EPMA de ligas Nb-Si-Ti tratadas termicamente a $1200^{\circ} \mathrm{C}$ : (a) dados em planilha eletrônica da composição (\% at.) de cada ponto medido da liga $24 \mathrm{HT}\left(\mathrm{Nb}_{11} \mathrm{Si}_{76} \mathrm{Ti}_{13} \%\right.$ at.); (b) diagrama de composições Ti X Si (\% at.) mostrando: a composição de cada ponto medido (\% at.);

- a locação da composição nominal das ligas $21 \mathrm{HT}$ ( $\mathrm{Nb}_{12} \mathrm{Si}_{60} \mathrm{Ti}_{28} \%$ at.), $23 \mathrm{HT}\left(\mathrm{Nb}_{17} \mathrm{Si}_{64} \mathrm{Ti}_{19} \%\right.$ at.) e 24 $\mathrm{HT}\left(\mathrm{Nb}_{11} \mathrm{Si}_{76} \mathrm{Ti}_{13} \%\right.$ at.); $\boldsymbol{\Delta}$ composição das fases identificadas (\% at.); $\Delta$ campo de equilíbrio trifásico.

(posições aproximadas).

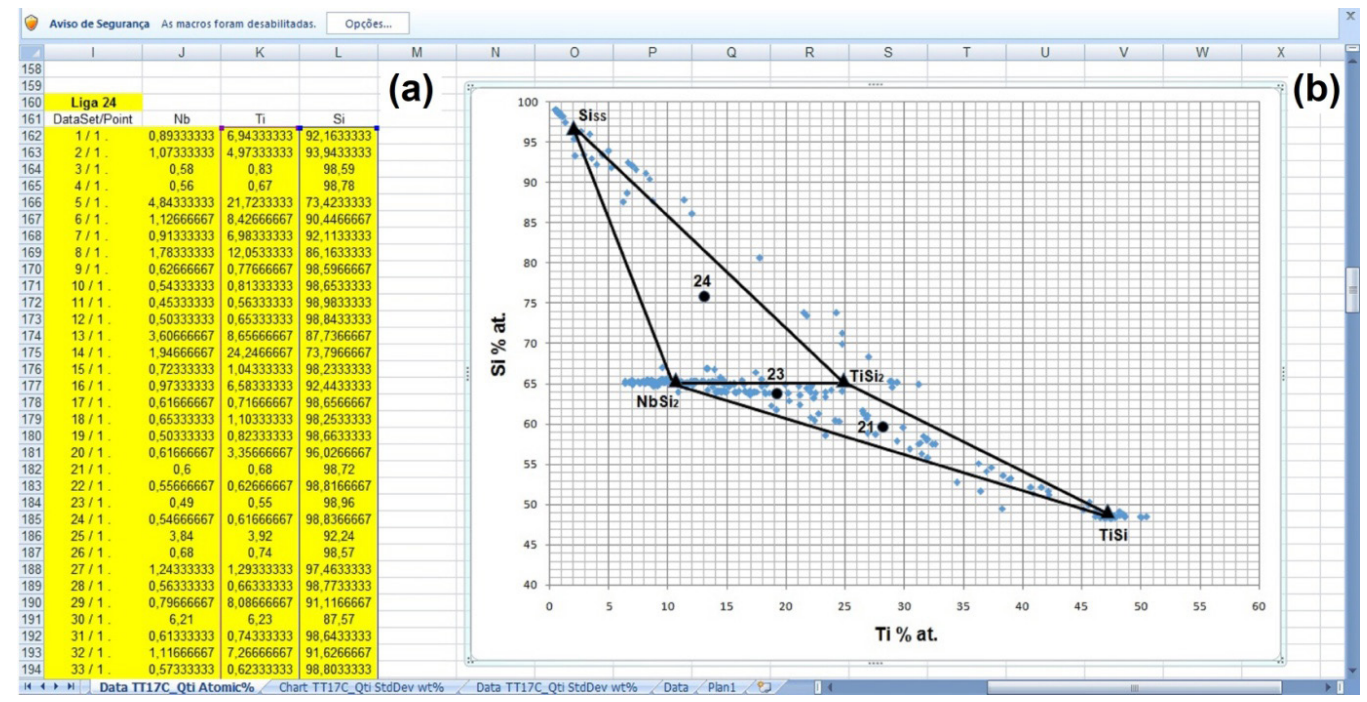

Fonte: Dos autores 2019

Assim, separados por fase todos os pontos medidos por EPMA, foi possível determinar a composição média de cada fase na liga $24 \mathrm{HT}\left(\mathrm{Nb}_{11} \mathrm{Si}_{76} \mathrm{Ti}_{13} \%\right.$ at.), a partir do cálculo da percentagem média de cada elemento químico ( $\mathrm{Nb}, \mathrm{Si}$ e Ti). Como exemplo, reunidos todos os pontos atribuídos para a fase $\mathrm{Si}_{\mathrm{SS}^{\prime}}$ conforme o critério na letra (c):

\begin{tabular}{cccc}
\hline Data set/Point & Nb & \\
\hline $9 / 1$. & 0,62666667 & 0,77666667 & 98,59666667 \\
$10 / 1$. & 0,54333333 & 0,81333333 & 98,65333333 \\
$11 / 1$. & 0,45333333 & 0,56333333 & 98,98333333 \\
etc. & & & \\
Média & 0,50000000 & 0,70000000 & 98,80000000 \\
\hline
\end{tabular}


Um diagrama de composições Ti X Si (\% at.) traçado a partir da planilha do programa, Figura $4 \mathrm{~b}$, permitiu fazer a locação de cada ponto medido ( $\downarrow$ na figura) e do ponto correspondente à composição média de cada fase $(\boldsymbol{\Lambda})$. Cada um desses pontos $(\boldsymbol{\Delta})$ corresponde a um vértice do triângulo que delimita a região de equilíbrio trifásico $\mathrm{Si}_{S \mathrm{~S}}-\mathrm{Nb}(\mathrm{Ti}) \mathrm{Si}_{2}-\mathrm{Ti}(\mathrm{Nb}) \mathrm{Si}_{2}$, na seção isotérmica a $1200{ }^{\circ} \mathrm{C}$ do sistema $\mathrm{Nb}$-Si-Ti. Para a definição final desse triângulo, também foram considerados os resultados de caracterização das ligas $21 \mathrm{HT}\left(\mathrm{Nb}_{12} \mathrm{Si}_{60} \mathrm{Ti}_{28} \%\right.$ at.) e $23 \mathrm{HT}\left(\mathrm{Nb}_{17} \mathrm{Si}_{64} \mathrm{Ti}_{19} \%\right.$ at.), especificamente no que concerne à composição das fases $\mathrm{Nb}(\mathrm{Ti}) \mathrm{Si}_{2}$ e $\mathrm{Ti}(\mathrm{Nb}) \mathrm{Si}_{2}$.

A quantidade percentual de cada fase presente na liga $24 \mathrm{HT}\left(\mathrm{Nb}_{11} \mathrm{Si}_{76} \mathrm{Ti}_{13} \%\right.$ at.), Tabela 2, foi determinada de duas formas: (1) através do refinamento dos dados de DRX pelo método Rietveld [31] com o programa FullProfSuite [32]; (2) a partir do conhecimento da composição da liga e das composições das fases que definem o campo de equilíbrio trifásico, utilizando-se a regra da alavanca [34]. Os valores obtidos em (1) e (2) apresentaram resultados semelhantes, um indicativo da correção das composições obtidas para as fases $\mathrm{Si}_{\mathrm{SS}^{\prime}} \mathrm{Nb}(\mathrm{Ti}) \mathrm{Si}_{2}$ e $\mathrm{Ti}(\mathrm{Nb}) \mathrm{Si}_{2}$.

Tabela 2 - Quantidade percentual em volume (\% vol.) das fases presentes na liga $24 \mathrm{HT}\left(\mathrm{Nb}_{11} \mathrm{Si}\right.$ ${ }_{76} \mathrm{Ti}_{13} \%$ at.): (a) cálculo pela regra da alavanca [34]; (b) resultados do refinamento dos dados de DRX $[31,32]$.

\begin{tabular}{cccc}
\hline Liga $\mathbf{1 2 0 0}{ }^{\circ} \mathrm{C} / \mathbf{7 2 0} \mathbf{h}$ & Fase & (a) \% vol. calculada & (b) \% vol. refinada \\
\hline $24 \mathrm{HT}$ & $\mathrm{Si}_{\mathrm{SS}}$ & 32,04 & 33,33 \\
$\left(\mathrm{Nb}_{11} \mathrm{Si}_{76} \mathrm{Ti}_{13} \%\right.$ at. $)$ & $\mathrm{Ti}(\mathrm{Nb}) \mathrm{Si}_{2}$ & 35,68 & \\
& $\mathrm{Nb}(\mathrm{Ti}) \mathrm{Si}_{2}$ & 32,28 & \\
\hline
\end{tabular}

Fonte: Dos autores 2019.

Exemplo 2 - Análise dos dados de EPMA da Liga $03 \mathrm{HT}\left(\mathrm{Cr}_{77} \mathrm{Hf}_{09} \mathrm{Nb}_{14} \%\right.$ at.), Figura $2 \mathrm{~b}$ e Tabela $1 \mathrm{~b}$, do sistema ternário $\mathrm{Cr}-\mathrm{Hf}-\mathrm{Nb}$.

Os resultados de DRX da Liga $03 \mathrm{HT}$, Figura 5a, mostraram a presença das fases $\mathrm{Cr}_{s S}$ ea( $\mathrm{Cr}_{2} \mathrm{Nb}$,$\mathrm{Cr}_{2} \mathrm{Hf}$ ). Observou-se picos intensos da fase $\mathrm{a}\left(\mathrm{Cr}_{2} \mathrm{Nb}, \mathrm{Cr}_{2} \mathrm{Hf}\right)$ enquanto a fase $\mathrm{Cr}_{s \mathrm{~s}}$ apresentou picos de menor intensidade. Tendo por base esses resultados, foi identificada na imagem MEV/IERE dessa liga, Figura $5 \mathrm{~b}$, uma microestrutura mista das fases $\mathrm{Cr}_{s S}$ ea $\left(\mathrm{Cr}_{2} \mathrm{Nb}, \mathrm{Cr}_{2} \mathrm{Hf}\right)$, o que caracteriza o campo de equilíbrio bifásico $\mathrm{Cr}_{\mathrm{Ss}}-\mathrm{a}\left(\mathrm{Cr}_{2} \mathrm{Nb}, \mathrm{Cr}_{2} \mathrm{Hf}\right)$. Observa-se também, uma correlação entre a grande quantidade de picos intensos da fasea $\left(\mathrm{Cr}_{2} \mathrm{Nb}, \mathrm{Cr}_{2} \mathrm{Hf}\right)$ no DRX e sua grande quantidade na micrografia, ao passo que a fase $\mathrm{Cr}_{\text {sS }}$ apresenta pequena quantidade na micrografia em consonância com o resultado de DRX. 
Utilização de ferramenta gráfica para a interpretação de dados de EPMA

na caracterização microestrutural de Ligas Ternárias à base de Nióbio

Figura 5 - Resultados de caracterização de liga $\mathrm{Cr}-\mathrm{Hf}-\mathrm{Nb}$ tratada termicamente a $1200^{\circ} \mathrm{C}$ : (a) DRX da liga $03 \mathrm{HT}\left(\mathrm{Cr}_{77} \mathrm{Hf}_{09} \mathrm{Nb}_{14}\right)$; (b) imagem MEV/IERE da liga $03 \mathrm{HT}\left(\mathrm{Cr}_{77} \mathrm{Hf}_{09} \mathrm{Nb}_{14}\right)$.
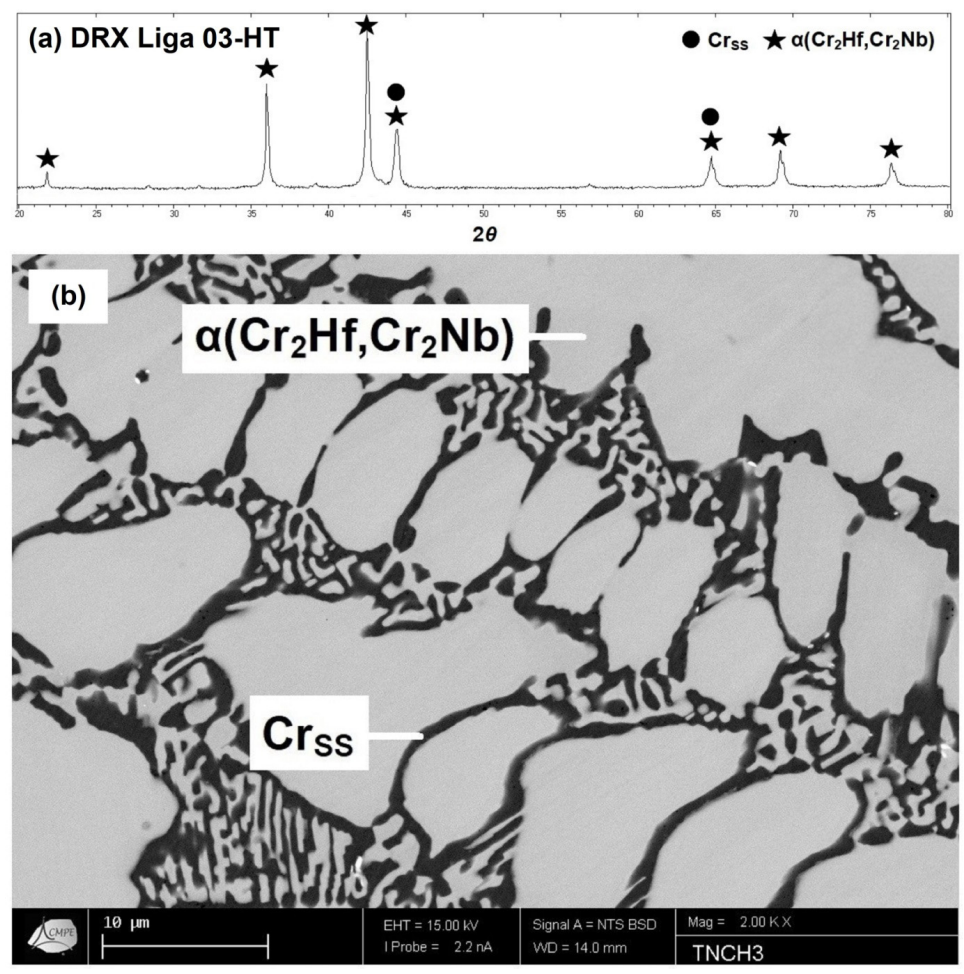

Fonte: autor.

Os resultados de EPMA da Liga $03 \mathrm{HT}\left(\mathrm{Cr}_{77} \mathrm{Hf}_{09} \mathrm{Nb}_{14} \%\right.$ at.) foram lançados numa planilha eletrônica, como visto parcialmente na Figura 6a. Cada ponto medido traz sua composição em \% at. tanto para o $\mathrm{Cr}$ quanto para o $\mathrm{Nb}$ e o $\mathrm{Hf}$. Como exemplo, o ponto identificado como 1/1. mostra a seguinte composição em \% at.: $\mathrm{Cr}$ 68,6465, Nb 18,3907 e Hf 12,9648.

A partir dos resultados de EPMA lançados na planilha eletrônica, cada ponto medido foi correlacionado a uma das duas fases identificadas por DRX e em MEV, conforme atendimento a um dos seguintes critérios:

(a) 0 ponto medido está na fase $\mathrm{a}\left(\mathrm{Cr}_{2} \mathrm{Nb}, \mathrm{Cr}_{2} \mathrm{Hf}\right)$ se apresenta: $66,7 \%$ at. $\mathrm{Cr}$ (até2 und. \% at. para $+/-), \Sigma(\mathrm{Nb}, \mathrm{Hf}) 33,3 \%$ at. (até 2 und. \% at. para $+/-$ );

(b) $O$ ponto medido está fase $\mathrm{Cr}_{\text {sS }}$ se apresenta: > 98,5\% at. $\mathrm{Cr}, \Sigma(\mathrm{Nb}, \mathrm{Hf})$ 1,5\% at.

Assim sendo, o ponto identificado como 1/1., no exemplo, conforme sua composição em \% at. preenche os critérios estabelecidos na letra (a) e, portanto, corresponde à fase $\mathrm{a}\left(\mathrm{Cr}_{2} \mathrm{Nb}, \mathrm{Cr}_{2} \mathrm{Hf}\right)$.

Assim, separados por fase todos os pontos medidos por EPMA, foi possível determinar a composição média de cada fase na liga $03 \mathrm{HT}\left(\mathrm{Cr}_{77} \mathrm{Hf}_{09} \mathrm{Nb}_{14} \%\right.$ at.), a partir do cálculo da percentagem média de cada elemento químico ( $\mathrm{Cr}, \mathrm{Nb}$ e Hf). $\mathrm{O}$ diagrama de composições $\mathrm{Nb}$ X Hf (\% at.) traçado a partir da planilha do programa, Figura 6b, permitiu fazer a locação de cada ponto medido ( $\downarrow$ na figura) e do ponto correspondente à composição média de cada fase $(\boldsymbol{\Delta})$. Cada um desses pontos $(\boldsymbol{\Lambda})$ corresponde a uma extremidade datieline[34] na região de equilíbrio bifásico $\mathrm{Cr}_{\mathrm{ss}}-\mathrm{a}\left(\mathrm{Cr}_{2} \mathrm{Nb}_{2} \mathrm{Cr}_{2} \mathrm{Hf}\right)$ na seção isotérmica a $1200^{\circ} \mathrm{C}$ do sistema $\mathrm{Cr}-\mathrm{Hf}-\mathrm{Nb}$. 
A quantidade percentual de cada fase presente na liga $03 \mathrm{HT}\left(\mathrm{Cr}_{77} \mathrm{Hf}_{09} \mathrm{Nb}_{14} \%\right.$ at.), Tabela 3, foi determinada através do refinamento dos dados de DRX [31,32] e também a partir do conhecimento da composição da liga e das composições das fases que definem o campo de equilíbrio bifásico, utilizando-se a regra da alavanca [34]. Os valores obtidos apresentaram resultados semelhantes, um indicativo da correção das composições obtidas para as fases $\mathrm{Cr}_{s S}$ e a $\left(\mathrm{Cr}_{2} \mathrm{Nb}_{2} \mathrm{Cr}_{2} \mathrm{Hf}\right)$.

Figura 6 - Resultados de EPMA da liga03 $\mathrm{HT}\left(\mathrm{Cr}_{77} \mathrm{Hf}_{09} \mathrm{Nb}_{14}\right)$ tratada termicamente a $1200^{\circ} \mathrm{C}$ : (a) dados em planilha eletrônica da composição (\% at.) de cada ponto analisado; (b) diagrama de composição $\mathrm{Nb}$ X Hf (\% at.) mostrando: a composição de cada ponto analisado (\% at.); • a locação da composição nominal da liga; $\boldsymbol{\Lambda}$ a composição da fase (\% at.); $\mathbf{\Lambda}---\boldsymbol{\Delta}$ a tieline. (posições aproximadas).

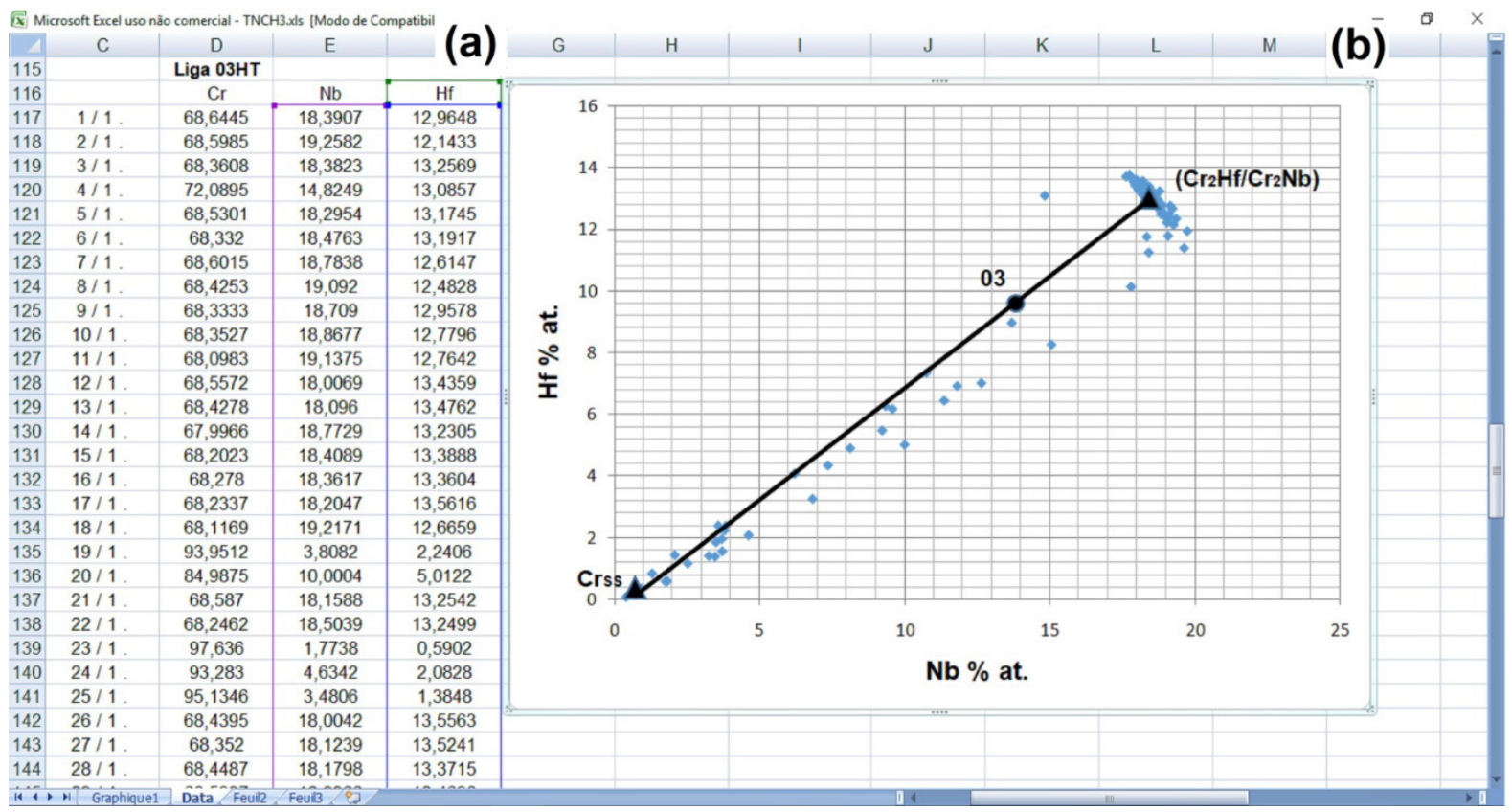

Fonte: Dos autores 2019.

Tabela 3 - Quantidade percentual em volume (\% vol.) das fases presentes na liga $03 \mathrm{HT}\left(\mathrm{Cr}_{77} \mathrm{Hf}_{09} \mathrm{Nb}_{14}\right.$ $\%$ at.): (a) cálculo pela regra da alavanca [34]; (b) resultados do refinamento dos dados de DRX [31,32].

\begin{tabular}{cccc}
\hline Liga $1200{ }^{\circ} \mathrm{C} 720 \mathrm{~h}$ & Fase & (a) \% vol. calculada & (b) \% vol. refinada \\
\hline $03 \mathrm{HT}$ & $\mathrm{Cr}_{S S}$ & 26,14 & 25,00 \\
$\left(\mathrm{Cr}_{77} \mathrm{Hf}_{09} \mathrm{Nb}_{14} \%\right.$ at. $)$ & $\mathrm{a}\left(\mathrm{Cr}_{2} \mathrm{Nb}_{2} \mathrm{Cr}{ }_{2} \mathrm{Hf}\right)$ & 73,86 & 75,00 \\
\hline
\end{tabular}

Fonte: Dos autores 2019.

\section{DISCUSSÃO DOS RESULTADOS}

A proposta elaborada por Gigolotti et al. [15] para a seção isotérmica a $1200^{\circ} \mathrm{C}$ do sistema $\mathrm{Nb}$ Si-Ti, Figura 2a e Tabela 1a, mostrou concordância com todos os campos de equilíbrio trifásico também definidos no trabalho experimental de Zhao et al. [24], Figura $1 \mathrm{~b}$, ou seja, a Nb(Ti) ${ }_{5} \mathrm{Si}_{3}-(\mathrm{Nb}, \mathrm{Ti})_{3} \mathrm{Si}-(\mathrm{Nb}, \mathrm{Ti})$ $\left.s^{\prime} \mathrm{Ti}^{\prime} \mathrm{Nb}\right)_{5} \mathrm{Si}_{3}-(\mathrm{Nb}, \mathrm{Ti})_{3} \mathrm{Si}-(\mathrm{Nb}, \mathrm{Ti})_{s s^{\prime}} \mathrm{aNb}(\mathrm{Ti})_{5} \mathrm{Si}_{3}-(\mathrm{Nb}, \mathrm{Ti})_{3} \mathrm{Si}-\mathrm{Ti}(\mathrm{Nb})_{5} \mathrm{Si}_{3^{\prime}}$ aNb $(\mathrm{Ti})_{5} \mathrm{Si}_{3}-\mathrm{Ti}(\mathrm{Nb})_{5} \mathrm{Si}_{4}-\mathrm{Ti}(\mathrm{Nb})_{5} \mathrm{Si}_{3^{\prime}}$ $\mathrm{aNb}_{5}(\mathrm{Ti}) \mathrm{Si}_{3}-\mathrm{Ti}(\mathrm{Nb})_{5} \mathrm{Si}_{4}-\mathrm{Nb}(\mathrm{Ti}) \mathrm{Si}_{2}, \mathrm{Ti}(\mathrm{Nb}) \mathrm{Si}-\mathrm{Ti}(\mathrm{Nb})_{5} \mathrm{Si}_{4}-\mathrm{Nb}(\mathrm{Ti}) \mathrm{Si}_{2}, \mathrm{Ti}_{(\mathrm{Nb}) S i}-\mathrm{Ti}(\mathrm{Nb}) \mathrm{Si}_{2}-\mathrm{Nb}(\mathrm{Ti}) \mathrm{Si}_{2}$ e $\mathrm{Si}_{\mathrm{SS}}-\mathrm{Ti}(\mathrm{Nb})$ 
Utilização de ferramenta gráfica para a interpretação de dados de EPMA

na caracterização microestrutural de Ligas Ternárias à base de Nióbio

$\mathrm{Si}_{2}-\mathrm{Nb}(\mathrm{Ti}) \mathrm{Si}_{2^{\prime}}$ mas com acentuadas diferenças nas composições das fases que os formam e, ainda, na faixa de solubilidade de $\mathrm{Si}$ nas fases aNb$\left(\mathrm{Ti}_{5}\right)_{5} \mathrm{Si}_{3^{\prime}} \mathrm{Ti}(\mathrm{Nb})_{5} \mathrm{Si}_{3^{\prime}} \mathrm{Ti}(\mathrm{Nb})_{5} \mathrm{Si}_{4^{\prime}} \mathrm{Nb}(\mathrm{Ti}) \mathrm{Si}_{2^{\prime}}, \mathrm{Ti}(\mathrm{Nb}) \mathrm{Si}$ e $\mathrm{Ti}(\mathrm{Nb}) \mathrm{Si}_{2}$.

Por sua vez, na comparação entre a proposta elaborada por Gigolotti et al. [15] e a proposta de Wang et al. [23], na região rica em $\mathrm{Nb}$-Ti, concordam na existência dos campos de equilíbrio trifásico $\mathrm{aNb}(\mathrm{Ti})_{5} \mathrm{Si}_{3}-(\mathrm{Nb}, \mathrm{Ti})_{3} \mathrm{Si}-(\mathrm{Nb}, \mathrm{Ti})_{S^{\prime}} \mathrm{Ti}(\mathrm{Nb})_{5} \mathrm{Si}_{3}-(\mathrm{Nb}, \mathrm{Ti})_{3} \mathrm{Si}-(\mathrm{Nb}, \mathrm{Ti})_{S \mathrm{~S}}$ e a Nb(Ti) $)_{5} \mathrm{Si}_{3}-(\mathrm{Nb}, \mathrm{Ti})_{3} \mathrm{Si}-\mathrm{Ti}(\mathrm{Nb})_{5} \mathrm{Si}_{3^{\prime}}$ enquanto na região rica em $\mathrm{Si}$, concordam no campoSi $\mathrm{SS}_{3 \mathrm{Ti}}-\mathrm{Ti}(\mathrm{Nb}) \mathrm{Si}_{2}-\mathrm{Nb}(\mathrm{Ti}) \mathrm{Si}_{2}$, mas com marcantes discrepâncias na composição das fases que os formam. Na região intermediária, apresentam concordância na existência dos campos aNb(Ti) ${ }_{5} \mathrm{Si}_{3}-\mathrm{Ti}(\mathrm{Nb})_{5} \mathrm{Si}_{4}-\mathrm{Ti}(\mathrm{Nb})_{5} \mathrm{Si}_{3}$ e $\mathrm{aNb}_{5}(\mathrm{Ti}) \mathrm{Si}_{3}-\mathrm{Ti}(\mathrm{Nb})_{5} \mathrm{Si}_{4}-\mathrm{Nb}(\mathrm{Ti}) \mathrm{Si}_{2^{\prime}}$ porém Wang et al. [23] definiram os campos $\mathrm{aNb}_{5}(\mathrm{Ti}) \mathrm{Si}_{3}-\mathrm{Ti}(\mathrm{Nb})_{5} \mathrm{Si}_{4}-\mathrm{Nb}(\mathrm{Ti}) \mathrm{Si}_{2}$ e $\mathrm{Ti}(\mathrm{Nb}) \mathrm{Si}_{-}-\mathrm{aNb}_{5}(\mathrm{Ti}) \mathrm{Si}_{3}-\mathrm{Ti}(\mathrm{Nb})_{5} \mathrm{Si}_{4}$, não reconhecidos por Gigolotti et al. [15].

No tocante à proposta elaborada por Gigolotti et al. [16] para a seção isotérmica a $1200{ }^{\circ} \mathrm{C}$ do sistema Cr-Hf-Nb, Figura $2 \mathrm{~b}$ e Tabela $1 \mathrm{~b}$, observou-se boa concordância com a proposta de Fujita et al. [28] na região rica em $\mathrm{Cr}$ com relação à existência e à extensão do campo de equilíbrio bifásico $\mathrm{Cr}_{\mathrm{SS}}{ }^{-}$ $\mathrm{a}\left(\mathrm{Cr}_{2} \mathrm{Nb}, \mathrm{Cr}_{2} \mathrm{Hf}\right)$ e quanto à existência e à faixa de solubilidade daseção pseudobináriaa $\left(\mathrm{Cr}_{2} \mathrm{Nb}_{1} \mathrm{Cr}_{2} \mathrm{Hf}\right)$, devendo-se destacar a proposta em primeira mãopor Gigolotti et al. [16], na região intermediária e na região rica em $\mathrm{Hf}-\mathrm{Nb}$ do sistema, da existência do campo trifásico $\mathrm{aHf}_{S s}-\mathrm{a}\left(\mathrm{Cr}_{2} \mathrm{Nb}_{2} \mathrm{Cr}_{2} \mathrm{Hf}\right)-(\beta \mathrm{Hf}, \mathrm{Nb})_{s S}$ e dos campos bifásicosaHf $s_{s s^{-}}(\beta \mathrm{Hf}, \mathrm{Nb})_{s s^{\prime}} a_{\mathrm{HH}}-\mathrm{a}\left(\mathrm{Cr}_{2} \mathrm{Nb}, \mathrm{Cr}_{2} \mathrm{Hf}\right)$ e $(\beta \mathrm{Hf}, \mathrm{Nb})_{s s}-\mathrm{a}\left(\mathrm{Cr}_{2} \mathrm{Nb}_{2} \mathrm{Cr}_{2} \mathrm{Hf}\right)$.

O grande transporte de massa que ocorreu entre as fases, a evolução microestrutural e as alterações nas relações de fases observados nas ligas tratadas termicamente, em comparação com essas ligas no estado bruto de fusão (as cast), permitiram uma definição confiável das relaçõesde fases na seção isotérmica a $1200^{\circ} \mathrm{C}$ em ambos os sistemas, Nb-Si-Ti[15] e Cr-Hf-Nb [16] e demonstraram a efetividade de serem utilizadas ligas composicionalmente selecionadas e fundidas para obter o equilíbrio termodinâmico durante tratamento térmico adequado.

Além disso, especificamente no estudo do sistema $\mathrm{Nb}$-Si-Ti, a combinação dos resultados de Gigolotti et al. [15] com os resultados obtidos por Zhao et al. [24],que usou pares de difusão, contribuíram para a solidez da definição da seção isotérmica a $1200^{\circ} \mathrm{C}$ do sistema. $\mathrm{O}$ uso de ligas fundidas, com composições particularmente selecionadas, conforme Kodentsov et al. [36], permite uma avaliação mais acurada de relações de fases, especialmentepara a determinação do equilíbrio de fases em seções isotérmicas em temperaturas não muito acima de $50 \%$ da temperatura média dos pontos de fusão dos elementos químicos envolvidos e dos compostos formados por reação congruente, como é o caso de $\mathrm{Nb}_{5} \mathrm{Si}_{3}, \mathrm{Ti}_{5} \mathrm{Si}_{3^{\prime}} \mathrm{Cr}_{2} \mathrm{Hf}$ e $\mathrm{Cr}_{2} \mathrm{Nb}$. Isso ficou claro no transporte de massa verificado nas ligas estudadas, durante o tratamento térmico. $\mathrm{O}$ uso do método de pares de difusão para o estudo de diagramas de fases carrega uma forte possibilidade de distorção do equilíbrio de fases e de verificação de sua estabilidade.

\section{CONCLUSÃO}

A precisão dos dados obtidos por EPMA combinados com resultados de DRX e imagens de MEV oferecem uma análise bastante confiável das relações de fases em seções isotérmicas de sistemas ternários à base de nióbio e outros metais refratários. Contudo, a complexa tarefa de analisar a grande quantidade de dados de EPMA pode ser facilitada com a utilização de uma ferramenta gráfica que consolide esses dados em diagramas. 0 presente trabalho apresentou exemplos desse tipo de análise de ligas metálicas, que permitiram a elaboração de propostas para as seções isotérmicas a $1200^{\circ} \mathrm{C}$ dos sistemas ternários $\mathrm{Nb}$-Si-Ti e $\mathrm{Cr}-\mathrm{Hf}-\mathrm{Nb}$. Na seção isotérmica a $1200{ }^{\circ} \mathrm{C}$ do sistema $\mathrm{Nb}$-Si-Ti ficaram definidos os campos de equilíbrio trifásico a $\mathrm{Nb}(\mathrm{Ti})_{5} \mathrm{Si}_{3}-(\mathrm{Nb}, \mathrm{Ti})_{3} \mathrm{Si}-(\mathrm{Nb}, \mathrm{Ti})_{S^{\prime}}, \mathrm{Ti}(\mathrm{Nb})_{5} \mathrm{Si}_{3}-(\mathrm{Nb}, \mathrm{Ti})_{3} \mathrm{Si}-(\mathrm{Nb}, \mathrm{Ti})$ $s^{\prime}, \mathrm{aNb}(\mathrm{Ti})_{5} \mathrm{Si}_{3}-(\mathrm{Nb}, \mathrm{Ti})_{3} \mathrm{Si}-\mathrm{Ti}(\mathrm{Nb})_{5} \mathrm{Si}_{3^{\prime}}$ aNb(Ti) $)_{5} \mathrm{Si}_{3}-\mathrm{Ti}(\mathrm{Nb})_{5} \mathrm{Si}_{4}-\mathrm{Ti}(\mathrm{Nb})_{5} \mathrm{Si}_{3^{\prime}} \mathrm{aNb}_{5}\left(\mathrm{Ti}^{\prime} \mathrm{Si}_{3}-\mathrm{Ti}(\mathrm{Nb})_{5} \mathrm{Si}_{4}-\mathrm{Nb}\left(\mathrm{Ti}^{\prime} \mathrm{Si}_{2^{\prime}}\right.\right.$ 
$\mathrm{Ti}(\mathrm{Nb}) \mathrm{Si}-\mathrm{Ti}(\mathrm{Nb})_{5} \mathrm{Si}_{4}-\mathrm{Nb}(\mathrm{Ti}) \mathrm{Si}_{2}, \mathrm{Ti}(\mathrm{Nb}) \mathrm{Si}-\mathrm{Ti}(\mathrm{Nb}) \mathrm{Si}_{2}-\mathrm{Nb}(\mathrm{Ti}) \mathrm{Si}_{2}$ e $\mathrm{Si}_{s s}-\mathrm{Ti}(\mathrm{Nb}) \mathrm{Si}_{2}-\mathrm{Nb}(\mathrm{Ti}) \mathrm{Si}_{2}$. Enquanto, na seção isotérmica a $1200^{\circ} \mathrm{C}$ do sistema $\mathrm{Cr}-\mathrm{Hf}-\mathrm{Nb}$, ficaram definidos o campo de equilíbrio trifásico $\mathrm{aHf}_{\mathrm{ss}}-\mathrm{a}(\mathrm{Cr}-$ $\left.{ }_{2} \mathrm{Nb}, \mathrm{Cr}_{2} \mathrm{Hf}\right)-(\beta \mathrm{Hf}, \mathrm{Nb})_{\mathrm{sS}^{\prime}}$ os campos bifásicos $\mathrm{Cr}_{\mathrm{ss}^{-}} \mathrm{a}\left(\mathrm{Cr}_{2} \mathrm{Nb}, \mathrm{Cr}_{2} \mathrm{Hf}\right), \mathrm{aHf}_{\mathrm{ss}}-(\beta \mathrm{Hf}, \mathrm{Nb})_{\mathrm{sS}^{\prime}} \mathrm{aHf}_{\mathrm{ss}_{\mathrm{s}}}-\mathrm{a}\left(\mathrm{Cr}_{2} \mathrm{Nb}, \mathrm{Cr}{ }_{2} \mathrm{Hf}\right) \mathrm{e}$ $(\beta \mathrm{Hf}, \mathrm{Nb})_{s s}-\mathrm{a}\left(\mathrm{Cr}_{2} \mathrm{Nb}, \mathrm{Cr}_{2} \mathrm{Hf}\right)$, além da seção pseudobináriaa $\left(\mathrm{Cr}_{2} \mathrm{Nb}, \mathrm{Cr}_{2} \mathrm{Hf}\right)$, com larga faixa de solubilidade.

\section{AGRADECIMENTOS}

Os autores agradecem ao CNPq (Conselho Nacional de Desenvolvimento Científico e Tecnológico), pelo suporte ao trabalho, por meio da bolsa de pós-doutorado 249666/2013-8 do programa "Ciência sem Fronteira".

\section{REFERÊNCIAS}

[1] KAYANI, S.H.; PARK, N.-K. Effect of $\mathrm{Cr}$ and $\mathrm{Nb}$ on the Phase Transformation and Pore Formation of Ti-Al Base Alloys. Journal of Alloys Compound, 708, p 308-315, 2017.

[2] POLOLOCK, T.M. Alloy Design for Aircraft Engines. Nature Materials, 15 (8), p 809-815, 2016.

[3] YAMAGUSHI, M.; INUI, H.; ITO, K. High-Temperature Structural Intermetallics. ActaMaterialia, 48 (1), p. 307-322, 2000.

[4] ZHAO, J-C.; WESTBROOK, J.H. Ultrahigh-Temperature Materials for Jet Engines. Materials Research Society (MRS) Bulletin, 28 (9), p. 622-630, 2003.

[5] DIMIDUK, D.M.; PEREPEZKO, J.H. Mo-Si-B Alloys: Developing a Revolutionary Turbine-Engine Material. Materials Research Society (MRS) Bulletin, 28 (9), p. 639-645, 2003.

[6] BEWLAY, B.P.; JACKSON, M.R.; ZHAO, J-C.; SUBRAMANIAN, P.R. A Review of Very-High-Temperature $\mathrm{Nb}$-Silicide-Based Composites. Metallurgical and Materials Transactions, 34 (A), p. 2043-2052, 2003.

[7] BEWLAY, B.P.; JACKSON, M.R.; GIGLIOTTI, M.F.X. Niobium Silicide High Temperature in-situ Composites. Intermetallic compounds, principles and practice: progress, 3, p. 541, 2002.

[8] BEWLAY, B.P.; JACKSON, M.R.; BRIANT, C.L. Deformation Mechanisms in Niobium Silicide-Based Composites - Final Report. Air Force Office of Scientific Research, p. 1-23, 2001.

[9] KENEL, C.; LEINENBACH, C. Influence of Nb and Mo on Microstructure Formation of Rapidly Solidified Ternary Ti-Al-(Nb,Mo) Alloys. Intermetallics, 69 (C), p. 82-89, 2016.

[10] VELLIOS, N.; TSAKIROPOULOS, P. The Role of Sn and Ti Additions in the Microstructure of Nb-18Si Base Alloys. Intermetallics, 15, p. 1518-1528, 2007.

[11] ZELENITSAS, K.; TSAKIROPOULOS, P. Effect of Al, Cr and Ta Additions on the Oxidation Behavior of $\mathrm{Nb}-\mathrm{Ti}-\mathrm{Si}$ In Situ Composites at $800^{\circ} \mathrm{C}$. Materials Science and Engineering - A, 416 (1-2), p. 269-280, 2006 .

[12] ZELENITSAS, K.; TSAKIROPOULOS, P. Study of the Role of Al and Cr Additions in the Microstructure of $\mathrm{Nb}$-Ti-Si In Situ Composites. Intermetallics, 13 (10), p. 1079-1095, 2005. 
Utilização de ferramenta gráfica para a interpretação de dados de EPMA

na caracterização microestrutural de Ligas Ternárias à base de Nióbio

[13] TAN, Y.H.; DU, Y. Isothermal Section of Cr-Ni-Ti System. Transactions of Nonferrous Metals Society of China, 17, p. 711-714, 2007.

[14] SU, L.; JIA, L.; WENG, J.; HONG, Z.; ZHOU, C.; ZHANG, H. Improvement in the Oxidation Resistance of $\mathrm{Nb}-\mathrm{Ti}-\mathrm{Si}-\mathrm{Cr}-\mathrm{Al}-\mathrm{Hf}$ Alloys Containing Alloyed Ge and B. Corrosion Science, 88, p. 460-465, 2014.

[15] GIGOLOTTI, J.C.J.; COELHO, G. C.; NUNES, C.A.; SUZUKI, P. A.; JOUBERT, J.-M. Experimental Evaluation of the $\mathrm{Nb}-\mathrm{Si}-\mathrm{Ti}$ System from As-Cast Alloys. Intermetallics, 82, p. 76-92, 2017.

[16] GIGOLOTTI, J.C.J.; NUNES, C.A.; COELHO, G. C.; JOUBERT, J.-M. Experimental Determination of the $\mathrm{Cr}-\mathrm{Hf}-\mathrm{Nb}$ System Liquidus Projection and $1200^{\circ} \mathrm{C}$ Isothermal Section. XVI Brazil MRS Meeting. Disponível em:http://sbpmat.org.br/16encontro/home/index.php. Acesso em: 22 fev. 2018.

[17] LIANG, H.; CHANG, Y.A. Thermodynamic Modeling of the Nb-Si-Ti Ternary System. Intermetallics, 7 (5), p. 561-570, 1999.

[18] CHEN, S.; YANG, Y.; CAO, W.; BEWLAY, B.P.; CHOU, K.-C.; CHANG, Y.A. Calculation of Two-Dimensional Sections of Liquidus Projections in Multicomponent Systems. Journal of Phase Equilibria and Diffusion, 29 (5), p. 390-397, 2008.

[19] GENG, T.; LI, C.; BAO, J.; ZHAO, X.; DU, Z.; GUO, C. Thermodynamic Assessment of the Nb-Si-Ti System. Intermetallics, 17 (5), p. 343-357, 2009.

[20] SUBRAMANIAN, P.R.; MENDIRATTA, M.G.; DIMIDUK, D.M. Microstructures and Mechanical Behavior of Nb-Ti Base Beta + Silicide Alloys. MRS Proceedings, 322, p. 491-502, 1994.

[21] BEWLAY, B.P.; JACKSON, M.R.; LIPSITT, H.A. The Nb-Ti-Si Ternary Phase Diagram: Evaluation of Liquid-Solid Phase Equilibria in Nb- and Ti-rich Alloys. Journal of Phase Equilibria, 18 (3), p. 264-278, 1997.

[22] BEWLAY, B.P.; JACKSON, M.R.; BISHOP, R.R. The Nb-Ti-Si Ternary Phase Diagram: Determination of Solid-State Phase Equilibria in Nb- and Ti-Rich Alloys. Journal of Phase Equilibria, 19 (6), p. 577$586,1998$.

[23] WANG, R.; LIU, C.; WANG, C.; JIN, Z. Determination of an Isothermal Section of the Nb-Ti-Si Ternary System at 1473 K. Rare Metal Materials and Engineering, 31 (5), p. 337-340, 2002.

[24] ZHAO, J.-C.; JACKSON, M.R.; PELUSO, L.A. Mapping of the Nb-Ti-Si Phase Diagram Using Diffusion Multiples. Materials Science and Engineering - A, 372 (1-2), p. 21-27, 2004.

[25] XU, H.; DU, Y.; CHEN, H.; HE, Y.; PAN, Z.; SCHUSTER, J.C.; WANG, R. Isothermal Section at $1000^{\circ} \mathrm{C}$ of the Nb-Ti-Si System. Journal of Alloys and Compounds, 394 (1-2), p. 235-239, 2005.

[26] BAO, J.; HUANG, Q.; TANG, L.; GENG, T.; ZHAO, X.; MA, C. Liquid-Solid Phase Equilibria of Nb-Si-Ti Ternary Alloys. Chinese Journal of Aeronautics, 21 (3), p. 275-280, 2008.

[27] LI, Y.; LI, C.-R.; DU, Z.-M.; GUO, C.-P.; ZHAO, X.-Q. As-cast Microstructures and Solidification Paths of the $\mathrm{Nb}-\mathrm{Si}-\mathrm{Ti}$ Ternary Alloys in $\mathrm{Nb}_{5} \mathrm{Si}_{3}-\mathrm{Ti}_{5} \mathrm{Si}_{3}$ Region. Rare Metals, 32 (5), p. 502-511, 2013. 
[28] FUJITA, M.; KANENO, Y.; TAKASUGI, T. Phase field and room-temperature mechanical properties of $\mathrm{C} 15$ Laves phase in $\mathrm{Nb}-\mathrm{Hf}-\mathrm{Cr}$ and $\mathrm{Nb}-\mathrm{Ta}-\mathrm{Cr}$ alloy systems. Journal of Alloys and Compounds, 424, 283-288, 2006.

[29] KRAUS, W.; NOLZE, G. PowderCell for Windows (version 2.4). Federal Institute for Materials research and testing, Berlin, 1999.

[30] VILLARS, P.; CALVERT, L.D. Pearsons's Handbook of Crystallographic Data for Intermetallic Phases ( $2^{\circ}$ Ed.). ASM International, 4, p. 4527-5263, 1990.

[31] RIETVELD, H.M. A profile refinement method for nuclear and magnetic structures. Journal of Applied Crystallography, 2 (b), p. 65-71, 1969.

[32] RODRIGUEZ-CARVAJAL, J. Recent Advances in Structure Determination by Neutron Powder Diffraction + FullProf. Physica B: CondensedMatter, 1993, 192 (1-2), p 55-56. Disponível em: http://www.ill.eu/ sites/fullprof/. Acesso em: 29 abr. 2015.

[33] CAMECA Science \&MetodologySolution. Introductionto EPMA. Disponível em: http://www.cameca. com/instruments-for-research/epma.aspx. Acesso em: 05 mar. 2018.

[34] RHINES, F.N. Phase Diagrams in Metallurgy - Their Development and Application. McGraw-Hill Company Inc., p. 126, 1956.

[35] SHIMADZU Analytical and Measuring Instruments. EPMA 1720/1720H. Disponível em: http:// www.shimadzu.com.br/analitica/produtos/surface/epma/epma-1720.shtml. Acesso em: 05 mar. 2018.

[36] KODENTSOV, A.A.; BASTIN, G.F.; LOO, F.J.J. VAN. The diffusion couple technique in phase diagram determination. Journal of Alloys and Compounds, 320 (2), p. 207-217, 2001. 